\title{
Indicadores de sustentabilidad para la pesquería de curvina golfina Cynoscion othonopterus en el Alto Golfo de California
}

\author{
Sustainability fishery indicator for Gulf corvina Cynoscion othonopterus in the Upper Gulf of California \\ Jaime E. Mendivil-Mendoza ${ }^{1 *}$, E. Alberto Aragón-Noriega ${ }^{1}$, José A. Arreola-

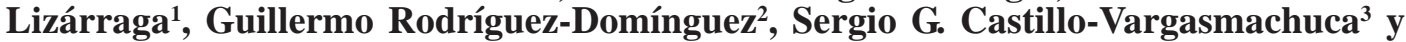 \\ Gilberto G. Ortega-Lizárraga ${ }^{4}$
}

${ }^{1}$ Centro de Investigaciones Biológicas del Noroeste, Unidad Sonora, km 2,35 Camino al Tular, Estero Bacochibampo, Guaymas, Sonora, 85454, México.*jaime_mendivil@hotmail.com

${ }^{2}$ Posgrado, Facultad de Ciencias del Mar, Universidad Autónoma de Sinaloa, Paseo Claussen s/n, Mazatlán, Sinaloa, 82000, México ${ }^{3}$ Posgrado en Ciencias Biológico Agropecuarias, Universidad Autónoma de Nayarit, Carretera Tepic-Compostela km 9 Xalisco, Nayarit, 63780, México

${ }^{4}$ Centro Regional de Investigación Pesquera Ensenada, Carretera Tijuana-Ensenada km 97,5 El Sauzal de Rodríguez, Ensenada, Baja California, 22760, México

\begin{abstract}
The Upper Gulf of California and Colorado River Delta is a zone which is immersed in an ecological, economic, political and social conflict. That is because of the Totoaba Totoaba macdonaldi illegal fishing and the Vaquita Phocoena sinus potential extinction. This issue has driven to ban all the region's fisheries with the exception of the Gulf corvina Cynoscion othonopterus which is the only of the finfish species with operating license. Therefore, the main objetive was to evaluate the fishery's health condition through the Froese sustainability indicators in Upper Gulf of California during 2008, 2013, 2014 and 2015 fishing seasons. The results showed that the Gulf corvina annual production ratio showed fluctuations, highlighting in the 2012-2016 period, when the whole fishing captures with the exception of 2014, surpassed the established quota by the National Institute of Fishery (Instituto Nacional de Pesca). The size-at-maturity were: $2008=L_{5}: 502 \mathrm{~mm}$ (IC= 490-514 mm), 2013= $L_{50}: 559 \mathrm{~mm}(\mathrm{IC}=544-576 \mathrm{~mm}), 2014=L_{50}: 499 \mathrm{~mm}(\mathrm{IC}=476-518 \mathrm{~mm})$, and $2015=L_{50}: 480 \mathrm{~mm}$ (IC= 445$510 \mathrm{~mm}$ ). Based on the sustainability indicators, it is demonstrated that the Gulf corvina fishery is directed towards the organisms of greater length (mega-spawners). Finally it is concluded that Gulf corvina currently shows problems of overexploitation and therefore its exploitation is not sustainable. This suggests the need to make adjustments and updates to current management measures.
\end{abstract}

Key words: Sustainability indicators, Cynoscion othonopterus, mega-spawners, optimum length, Upper Gulf of California

Resumen.- El Alto Golfo de California y Delta del Río Colorado es un área que se encuentra inmersa en un conflicto ecológico, económico, político y social debido a la pesca ilegal de totoaba Totoaba macdonaldi y la posible extinción de vaquita marina Phocoena sinus. Esto último ha provocado el cierre de todas las pesquerías en la región salvo la de curvina golfina Cynoscion othonopterus la cual es la única especie con permiso de explotación. Por lo tanto, el objetivo principal fue evaluar el estado de salud de la pesquería mediante los indicadores de sustentabilidad de Froese en el Alto Golfo de California durante las temporadas de pesca 2008, 2013, 2014 y 2015. Los resultados mostraron que la producción anual de curvina golfina presenta fluctuaciones, resaltando el periodo 2012-2016, donde las capturas totales con excepción de 2014, sobrepasaron la cuota de captura establecida por el Instituto Nacional de la Pesca. La longitud de madurez para los años estudiados fueron: $2008=L_{50}: 502 \mathrm{~mm}($ IC $=490-514 \mathrm{~mm}), 2013=L_{50}: 559 \mathrm{~mm}$ (IC= 544-576 mm), $2014=L_{50}: 499 \mathrm{~mm}($ IC= $476-518 \mathrm{~mm}$ ), y $2015=L_{50}: 480 \mathrm{~mm}($ IC $=445-510 \mathrm{~mm}$ ). Con base en los indicadores de sustentabilidad, se demuestra que la pesquería de curvina golfina está dirigida hacia los organismos de mayor longitud (mega-reproductores). Se concluye que actualmente la curvina golfina manifiesta problemas de sobreexplotación y por ende su aprovechamiento no es sustentable. Esto sugiere la necesidad realizar ajustes y actualizaciones a las actuales medidas de manejo.

Palabras clave: Indicadores de sustentabilidad, Cynoscion othonopterus, mega reproductores, longitud óptima, Alto Golfo de California 


\section{INTRODUCCIÓN}

Las pesquerías a nivel mundial constituyen una importante fuente de alimentos, empleo y generación de ingresos que impacta positivamente en el sustento familiar. Debido a lo anterior y gracias al desarrollo de los conocimientos y evolución de la dinámica de las pesquerías tenemos conciencia que los recursos pesqueros no son ilimitados, más bien son renovables y limitados (FAO 1995). Una forma directa de evaluar los recursos pesqueros es mediante el acompañamiento de la pesquería (Barbarino et al. 1998). Dicha información contribuye a mejorar el conocimiento de la biodiversidad y dinámica de los ecosistemas acuáticos, así como al establecimiento de líneas de base para la evaluación y manejo de pesquerías (Pauly 1983). Por ello, es importante tener un sistema de referencia, que ayude a mejorar el manejo de los recursos explotados y preservar la sustentabilidad de los organismos acuáticos (FAO 2000, García et al. 2000). Sobre esto, Rice \& Rochet (2005) mencionan que para lograr la sustentabilidad de las pesquerías, es necesario utilizar indicadores que proporcionen un mejor entendimiento del estado del 'stock'. En particular, la actividad pesquera en el Alto Golfo de California (AGC) ha sido singularmente problemática por tener una amplia dispersión espacial y una marcada disparidad social (Doode \& Wong 2001). Rodríguez-Quiroz et al. (2010) mencionan que uno de los principales recursos de escama para las comunidades del AGC ha sido la curvina golfina Cynoscion othonopterus (Jordan \& Gilbert, 1882). Actualmente la pesquería de $C$. othonopterus es la más importante en la región debido a que es la única especie con permiso de explotación decretado por el Gobierno de México (DOF 2015) ${ }^{1}$.

La restricción hacia las demás pesquerías se debe a los problemas ecológicos (modificación del hábitat y el riesgo de extinción de vaquita marina Phocoena sinus), económico (pesca ilegal de totoaba Totoaba macdonaldi), político-social (incumplimiento de la legislación ambiental debido a la pesca en la zona núcleo de la Reserva de la Biosfera del AGC (Campoy 1999) y conflictos con la comunidad indígena Cucapá, habitantes ancestrales de

${ }^{1}$ DOF 2015. Acuerdo por el que se suspende temporalmente la pesca comercial mediante el uso de redes de enmalle, cimbras y/o palangres operadas con embarcaciones menores, en el Norte del Golfo de California <http://www.dof.gob.mx/ nota_to_imagen_fs.php?codnota $=5388486 \&$ fecha $=10 / 04 /$ 2015\&cod_diario $=263621>$ la región, quienes demandan exclusividad en el aprovechamiento de recursos pesqueros (RománRodríguez \& Barrera-Guevara 2006).

La temporada de pesca de curvina golfina se restringe a una fecha de inicio variable durante febrero y finaliza el 30 de abril de cada año. Con el fin de aprovechar al máximo la pesquería cuidando la sustentabilidad, en agosto 2007 se emitió la Norma Oficial Mexicana para la pesca responsable de C. othonopterus (NOM-063-PESC-2005)2, que establece los términos y condiciones para el aprovechamiento del recurso. Asimismo, estos acuerdos se encuentran vigentes en el Plan de Manejo Pesquero de curvina golfina del Norte del Golfo de California. Algunos de los aspectos que establece la norma se evalúan en el presente estudio, con el fin de discutir cual ha sido su efectividad en la pesquería. Las medidas evaluadas fueron: longitud de madurez, cuotas y longitud de captura. Por otro lado, se propone actualizar la Norma Oficial y el Plan de Manejo Pesquero con los resultados obtenidos, así como incorporar el estimador de Froese (2004). Este método ayudará a determinar el grado de sobrepesca en la pesquería basándose en 3 indicadores pesqueros: (1) proporción de organismos maduros en la captura, (2) longitud óptima de captura y (3) proporción de mega-reproductores capturados. El objetivo general fue determinar el estado de la pesquería de curvina golfina del Alto Golfo de California mediante los indicadores de sustentabilidad de Froese (2004).

\section{Materiales y MÉtodos}

El área de estudio se localiza en el Alto Golfo de California, en la Reserva de la Biosfera del Alto Golfo de California y Delta del Rio Colorado (RBAGCyDRC en las coordenadas $31-32^{\circ} \mathrm{N}$ y $114-115^{\circ} \mathrm{W}$, decretada como Área Natural Protegida en 1993 por el gobierno mexicano. Cuenta con una superficie de 934.756 ha que comprende una zona núcleo de 164.779 ha y una zona de amortiguamiento de 769.976 ha (DOF 1993)

\footnotetext{
${ }^{2}$ NOM-063-PESC-2005. 2007. Pesca responsable de curvina golfina (Cynoscion othonopterus) en aguas de jurisdicción federal del Alto Golfo de California y Delta del Río Colorado. Especificaciones para su aprovechamiento. <http://sagarpa.gob.mx/normateca/ normateca2/52\%20NORMA\%200ficial\%20Mexicana\%20NOM063-PESC-2005\%20160807.pdf>

${ }^{3}$ DOF. 1993. Decreto por el que se declara área natural protegida con el carácter de Reserva de la Biosfera, la región conocida como Alto Golfo de California y Delta del Río Colorado, ubicada en aguas del Golfo de California y los municipios de Mexicali, B.C., de Puerto Peñasco y San Luis, Río Colorado, Sonora.
} 
Desarrollo de los muestreos y fuente de INFORMACIÓN

El trabajo de campo se realizó 3 días antes de las mareas vivas durante febrero a abril de 2008, 2013, 2014 y 2015. Se capturaron organismos con redes de enmalle de $200 \mathrm{~m}$ de longitud por $2 \mathrm{~m}$ de caída con 14,6 mm de luz de malla, siguiendo los criterios de Solana-Sansores et al. (2012). Los organismos fueron separados por sexo para posteriormente registrar el peso total mediante una balanza electrónica (UVD 500) con precisión de 0,1g, se midió la longitud total usando un ictiómetro graduado en milímetros colocando los especímenes de Cynoscion othonopterus sobre el costado y siguiendo una dirección paralela al eje céfalo-caudal del cuerpo.

Se utilizaron los datos de muestreos propios para comparar las longitudes promedio de captura de cada temporada de pesca, pero por razones logísticas no se realizaron muestreos durante 2007, 2011, 2012 y 2016 por lo que para estos años se utilizó la longitud promedio reportada por Erisman et al. (2014).

\section{CAPTURA ANUAL}

Los datos de captura anual (t) de peso eviscerado para los Estados de Sonora y Baja California durante los años 1994-2015 se obtuvieron de la página de monitoreo administrativo de curvina golfina ${ }^{4}$. Información más detallada de las capturas (mensuales, por marea o por Estado, localidad etc.) puede ser consultada en la página web antes mencionada. La fuente de información para la captura de 2016 en el Estado de Sonora se obtuvo de la página oficial del Instituto de Acuacultura del Estado de Sonora, O.P.D ${ }^{5}$.Para la captura de 2016 en Baja California se consultó el boletín estadístico de reporte de producción pesquera y acuícola de Baja California sistema, SIPESCA/ CONAPESCA ${ }^{6}$.

\section{CONDICIÓN REPRODUCTIVA}

El criterio utilizado para designar un organismo maduro e inmaduro se realizó mediante el criterio macroscópico basado en la escala morfocromática para peces de Nikolsky (1963), rediseñada por Acosta-Valenzuela (2010)

\footnotetext{
${ }^{4}<$ https://sites.google.com/site/monitoreocurvinabc/^^

${ }^{5}<$ https://www.iaes.gob.mx>

${ }^{6}<$ http://www.sepescabc.gob.mx/x/estadisticas/docs/

PRODUCCION_PESQUERA_Y_ACUICOLA_DE_BC_2016-

(PRELIMINAR_31JUL2016).pdf>
}

para C. othonopterus. Los peces que previamente habían sido separados por sexo, ahora se separaron en estadios de madurez (estadios I-III inmaduros, estadios IV y V maduros).

\section{LONGITUD DE MADUREZ}

Se usaron los datos de las temporadas 2008, 2013, 2014 y 2015 y el cálculo de la longitud de madurez $\left(L_{50}\right)$ se realizó obteniendo el porcentaje de individuos maduros por clase de longitud cada $50 \mathrm{~mm}$, y posteriormente, ajustando estos valores a un modelo logístico (King 1995) a través de la siguiente ecuación:

$$
p_{i}=\frac{1}{1+\exp ^{-\left(L_{i}+L_{50}\right) / a}}
$$

Dónde:

$P_{i}=$ porcentaje de madurez a la longitud $i$

$L_{i}=$ intervalo de longitud

$L_{50}=$ longitud del 50\% de madurez poblacional

$a=$ constante de ajuste

Mediante iteraciones, los parámetros del modelo fueron ajustados maximizando la función de verosimilitud binomial propuesta por Brouwer \& Griffiths (2005):

$L L(\phi \mid$ datos $)-\sum_{\mathrm{i}=1}^{\mathrm{n}}\left[m_{i} \cdot \ln \left(\frac{p_{i}}{1-p_{i}}\right)+n_{i} \cdot \ln \left(1-p_{i}\right)+\ln \left(\begin{array}{c}n_{i} \\ m_{i}\end{array}\right)\right]$

Dónde:

$n_{i}=$ número de total de organismos de la clase $i$

$m_{i}=$ número de organismos maduros en la clase $i$

\section{INTERVALOS DE CONFIANZA}

Se calcularon los intervalos de confianza para longitud de madurez al 95\% con base en los perfiles de máxima verosimilitud y Chi-cuadrado (Venzon \& Moolgavkar 1988). El intervalo de confianza fue definido como todos los valores de $\theta$ que satisfacen la siguiente desigualdad:

$$
2\left(L L(Y \mid \theta)-L\left(Y \mid \theta_{\text {best }}\right)\right)<x^{2} 1,1-\alpha
$$

Dónde:

$L\left[Y \mid \theta_{\text {best }}\right]=$ es la máxima verosimilitud del valor más probable de $\theta$.

$x^{2} 1,1-\alpha=$ es el valor de Chi-cuadrado con un grado de libertad al nivel de confianza $1-\alpha$. 
Así, el intervalo de confianza al 95\% de $\theta$ abarca todos los valores de $\theta$ que son dos veces la diferencia entre la máxima verosimilitud de un $\theta$ dado y la máxima verosimilitud de la mejor estimación de que sea menor que 3,84 (Haddon 2001).

\section{INDICADORES DE SUSTENTABILIDAD DE FROESE (2004)}

Para determinar la sustentabilidad de la pesquería de curvina golfina se utilizó el estimador propuesto por Froese (2004) el cual contempla 3 indicadores pesqueros los cuales son: organismos maduros, longitud óptima y mega-reproductores los cuales se describen con más detalle a continuación:

Indicador $1\left(L_{50}\right)$ : dejar que los organismos desoven, el objetivo sería que el $100 \%$ de las curvinas golfinas capturadas hayan desovado por lo menos una vez con el fin de mantener el stock y reclutamiento.

Indicador $2\left(L_{\text {opt }}\right)$ : dejar que los organismos crezcan, el objetivo es que el $100 \%$ de la captura de curvina golfina esté dentro del $\pm 10 \%$ de la longitud óptima $\left(L_{\text {opt }}\right)$.

Indicador 3: dejar que los mega-reproductores vivan, y se mide como el porcentaje de organismos más longevos y de grandes longitudes en las capturas. Estos peces están por encima del 10\% de la longitud óptima. El objetivo fue poner en práctica una estrategia de pesca para evitar capturar mega-reproductores (0\%). Si no existiera tal estrategia se permite capturar entre un $30-40 \%$ de mega- reproductores de la captura total. Si las capturas reflejan un 20\% de mega-reproductores sería motivo de alerta.

Para determinar la longitud óptima de curvina golfina se empleó la fórmula empírica propuesta por Froese \& Binohlan (2000).

$$
\log _{10} \mathrm{~L}_{\mathrm{opt}}=1,053 * \log _{10}\left(L_{50}\right)-0,0565
$$

Dónde:

$L_{50}=$ Longitud del $50 \%$ de madurez poblacional

$L_{\text {opt }}=$ Longitud correspondiente a la edad media cuando el $100 \%$ de los peces han desovado por lo menos una vez.

\section{Resultados}

Se presenta la longitud promedio anual desde 1997 hasta el 2016 obteniéndose una longitud mínima de 627 mm para 2003 y una longitud máxima de 703 mm para 1998. Durante el periodo 2008 a 2016 se presenta una tendencia negativa respecto a la longitud, asimismo, se observa una estandarización con respecto al error de los datos (Fig. 1). Cabe mencionar que los datos que no presentan desviación estándar fueron datos que se tomaron de la literatura. Las capturas anuales de peso eviscerado para los Estados de Sonora y Baja California muestran una captura máxima de 5.389 t en el 2016 y una mínima de 204 t en 1994. Las capturas presentaron un incremento exponencial de 1994 a 2002 para posteriormente disminuir drásticamente en 2003 y 2004. A partir de 2005 se vuelve a presentar una

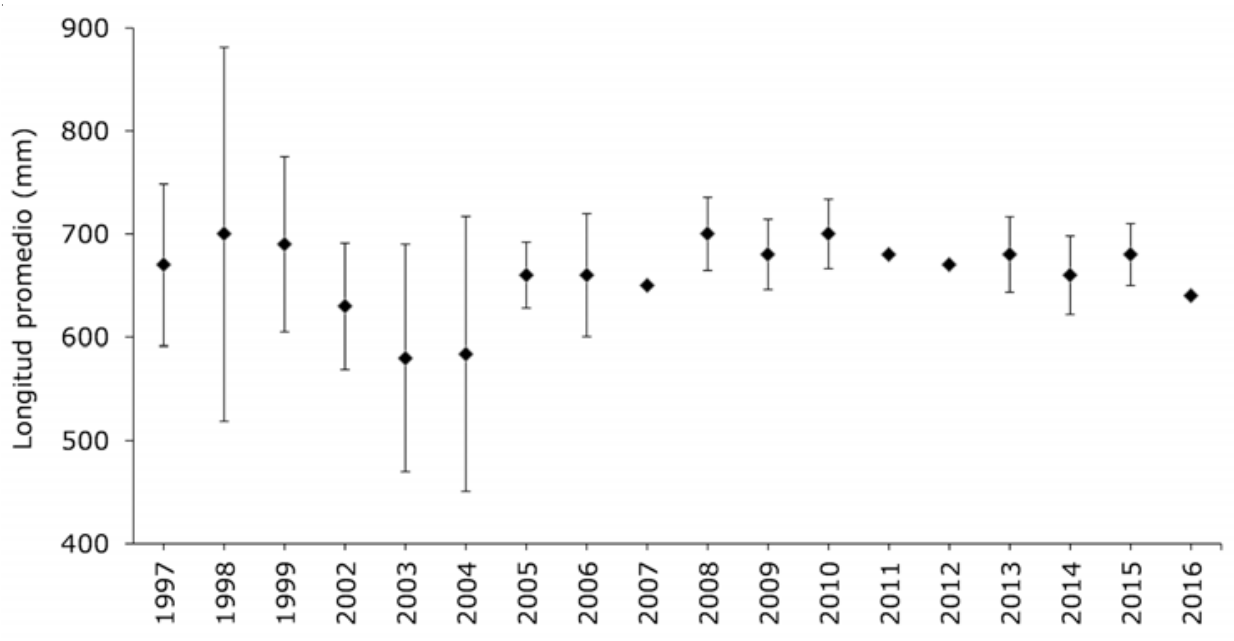

Figura 1. Variaciones de la composición de la longitud promedio de la pesquería de curvina golfina Cynoscion othonopterus para las temporadas 1997-2016 / Composition variations of the average length for the Gulf corvina Cynoscion othonopterus for the 1997-2016 seasons 


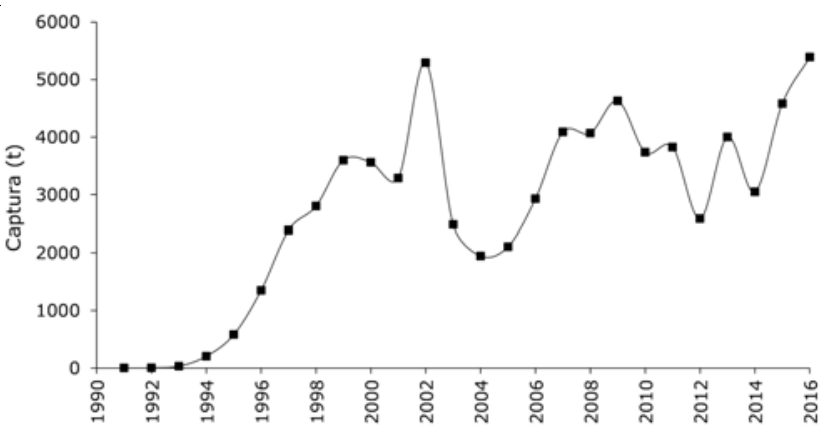

Figura 2. Producción de curvina golfina Cynoscion othonopterus en el Alto Golfo de California para los años 1997-2016 / Gulf corvina Cynoscion othonopterus production in the Upper Gulf of California for the 1997-2016 interval segunda tendencia positiva hasta el 2009. Las capturas para el periodo 2010 a 2014 fueron inestables ya que presentaron fluctuaciones tanto positivas como negativas, por último desde el 2015 a la fecha se presentó un marcado incremento (Fig. 2). En general las capturas de curvina golfina son inestables ya que han presentado a través de los años tendencias oscilatorias. Para estimar la longitud de madurez se analizaron un total de 1.314 hembras de las cuales 1.023 se encontraban maduras. La longitud mínima registrada de madurez fue 350 y la máxima de 950 mm. La distribución de longitud osciló entre 150 a $950 \mathrm{~mm}$, estando más representado por organismos de 600 a $850 \mathrm{~mm}$. La longitud de madurez $\left(L_{50}\right)$ para los años de estudio y respectivo intervalo de confianza mediante los perfiles de máxima verosimilitud fue: 2008 $=L_{50}: 502$ $\mathrm{mm}(\mathrm{IC}=490-514 \mathrm{~mm}), 2013=L_{50}: 559 \mathrm{~mm}(\mathrm{IC}=544-576$ $\mathrm{mm}$;), 2014= $L_{50}: 499 \mathrm{~mm}$ (IC= 476-518 mm), y 2015 $=L_{50}$ : $480 \mathrm{~mm}(\mathrm{IC}=445-510 \mathrm{~mm})$ (Figs. 3 y 4$)$.

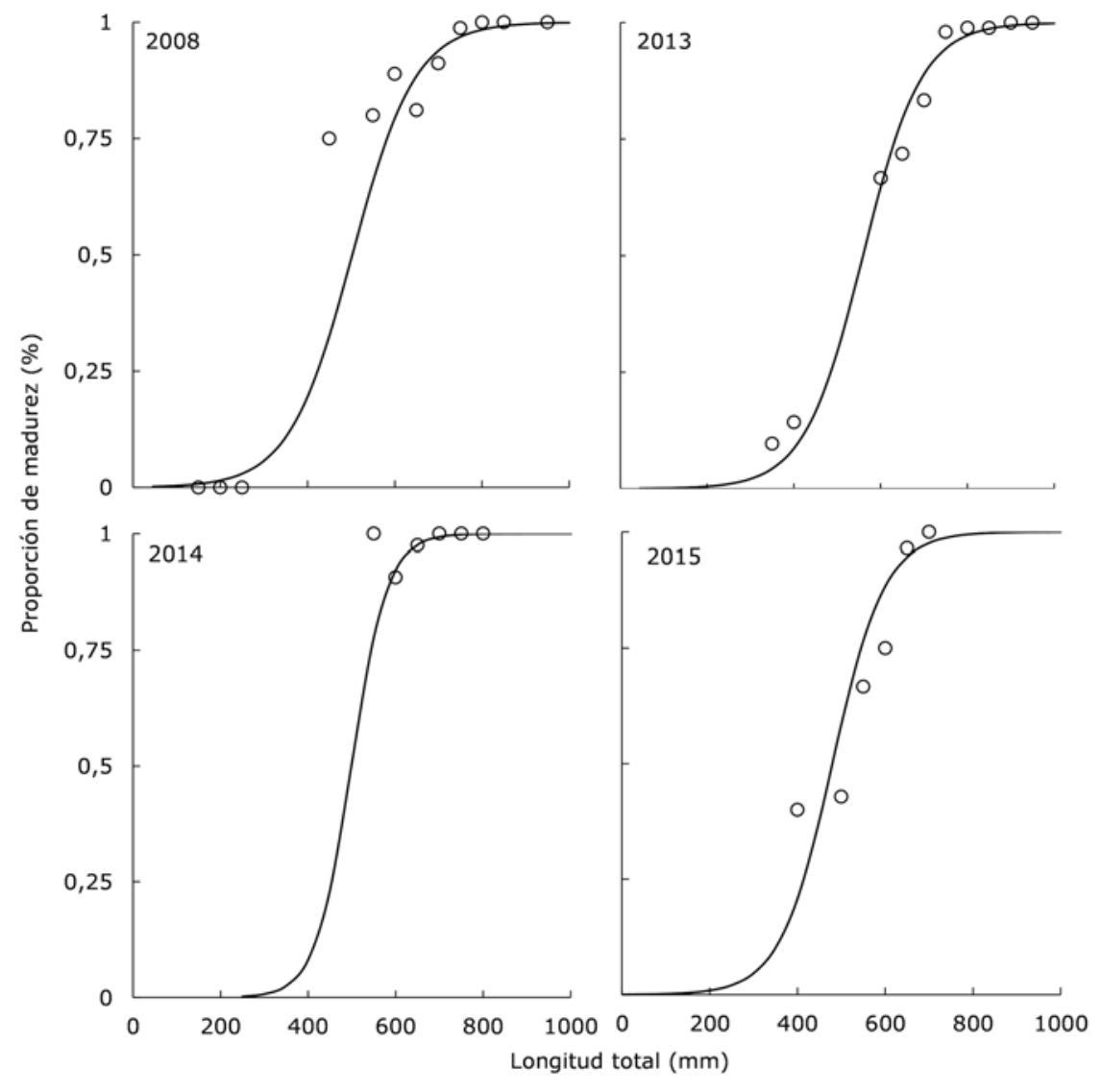

Figura 3. Curvas de la longitud de madurez para hembras de curvina golfina Cynoscion othonopterus capturadas en el Alto Golfo de California. Los círculos son datos observados mientras que la línea es la curva ajustada / Maturity length curves for female Gulf corvina Cynoscion othonopterus which were captured in Upper Gulf of California. The circles show the sampled data and the line is the adjustment curve 


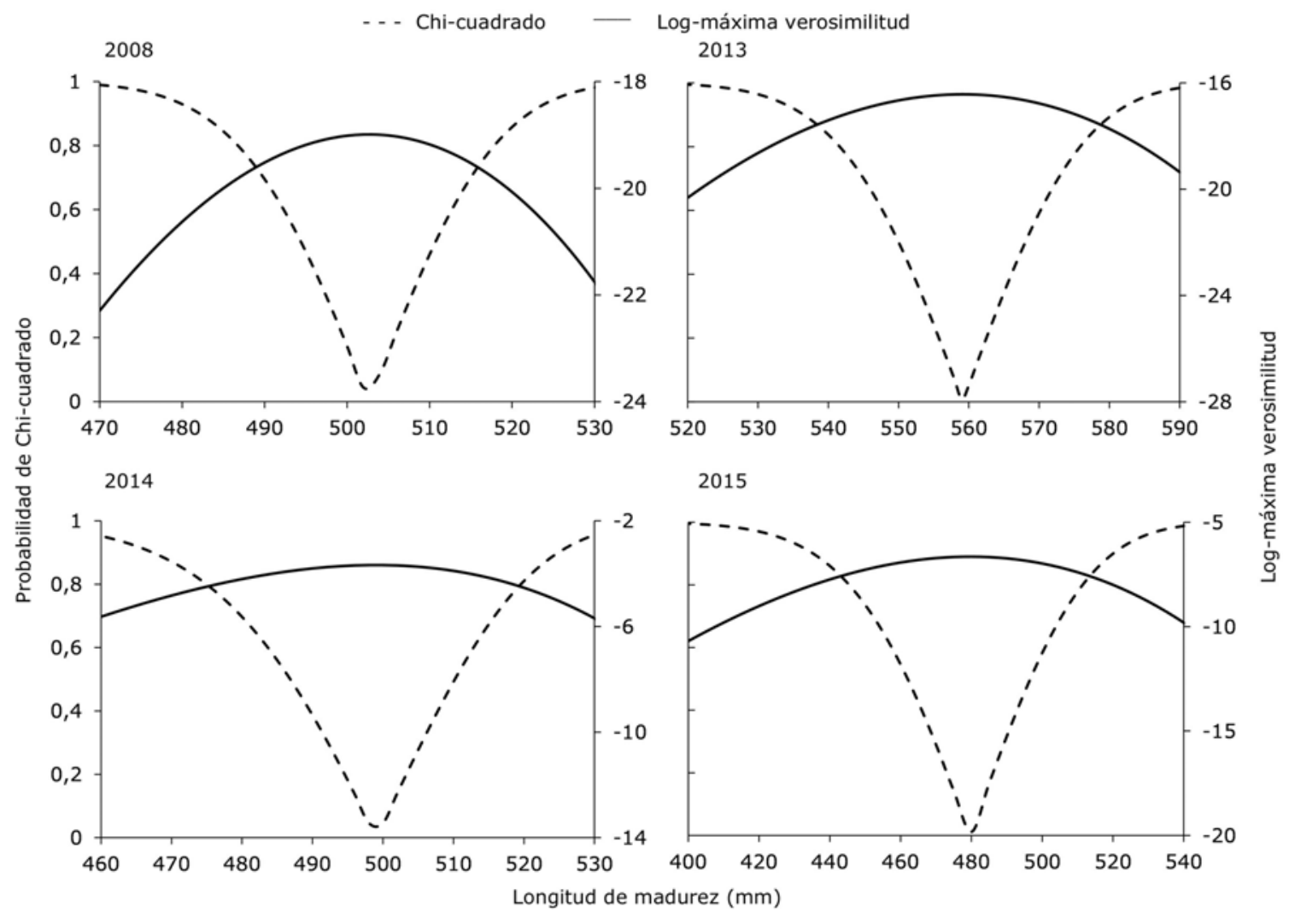

Figura 4. Intervalos de confianza mediante perfiles de verosimilitud para la longitud de madurez en hembras de Cynoscion othonopterus / Confidence interval by means of verisimilitude's profiles for female Gulf corvina Cynoscion othonopterus maturity length

Teniendo en cuenta los valores de $L_{50}$ se estimó la longitud óptima $\left(L_{o p}\right)$ mediante la fórmula empírica de Froese \& Binohlan (2000). Quedando representado para cada año de estudio una $L_{\text {opt }}$ de $613 \pm 10 \%$ para $2008,686 \pm 10 \%$ en $2013,609 \pm 10 \%$ en 2014 y $585 \mathrm{~mm} \pm 10 \%$ en 2015 . Una vez que se obtuvieron los resultados de $L_{50}$ y $L_{\text {opt }}$ se procedió a evaluar los indicadores de Froese (2004). Para el 2008 se analizaron 575 organismos y los indicadores muestran que el $16 \%$ fueron individuos inmaduros, $23 \%$ se encontraban dentro de la longitud óptima y el 61\% fueron mega-reproductores. Durante la temporada de pesca 2013 se analizaron 494 organismos de los cuales el 56\% fueron mega-reproductores, el 21\% estuvieron por debajo de la longitud de madurez y el 23\% estuvieron dentro de la longitud óptima. En la temporada 2014 se examinaron 163 organismos de los cuales el $22 \%$ fueron megareproductores, el $78 \%$ de los organismows se consideran estuvieron dentro de la longitud óptima y no se encontraron capturas de organismos por debajo de la longitud de madurez. Por último, en la campaña de pesca 2015 se analizaron 82 curvinas golfinas que estuvieron representadas por el 70\% por mega-reproductores, el 8\% fueron juveniles y el 22\% estuvieron dentro de la longitud óptima (Fig. 5).

\section{Discusión}

Determinar la longitud a la cual se capturan los organismos es de vital importancia para la conservación de las especies debido a que permite inferir si las capturas están respetando la longitud mínima de madurez y la luz de malla establecida para la explotación de los recursos. En la presente investigación se logró determinar que durante el periodo de pesca de 1997 al 2006 el promedio de longitud para Cynoscion othonopterus presentó una tendencia negativa así como un sesgo muy amplio de la desviación estándar coincidiendo con lo reportado por Erisman et al. (2014). Esto se debió a que los pescadores utilizaban 
A)

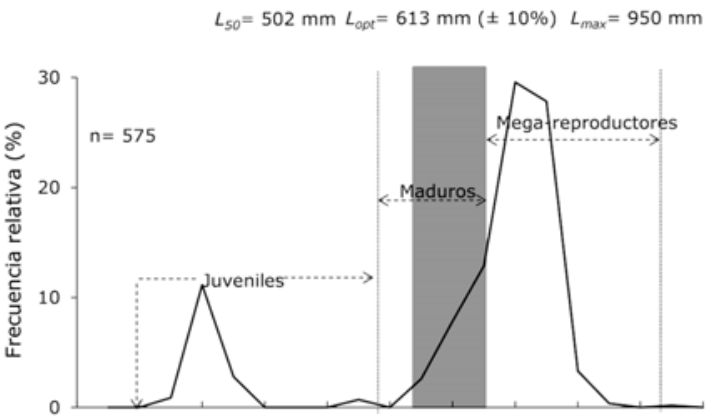

C)

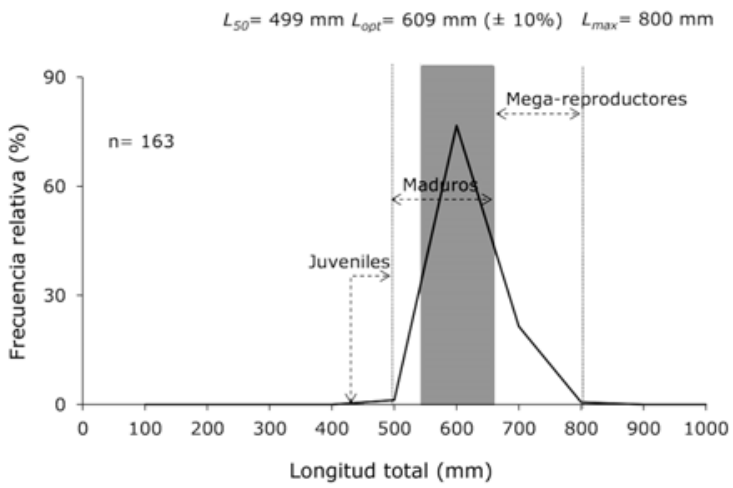

B)

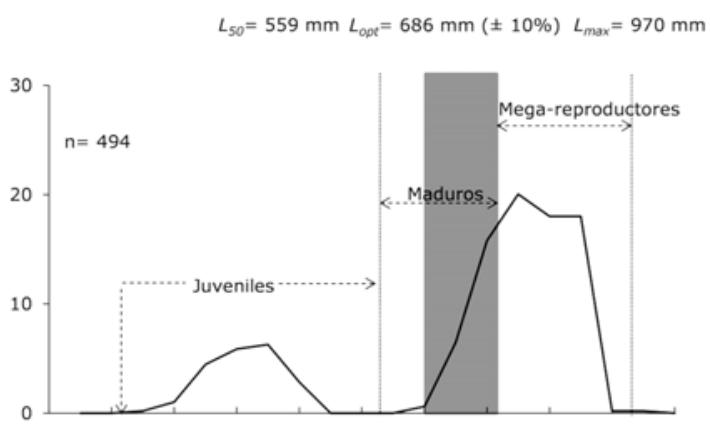

D)

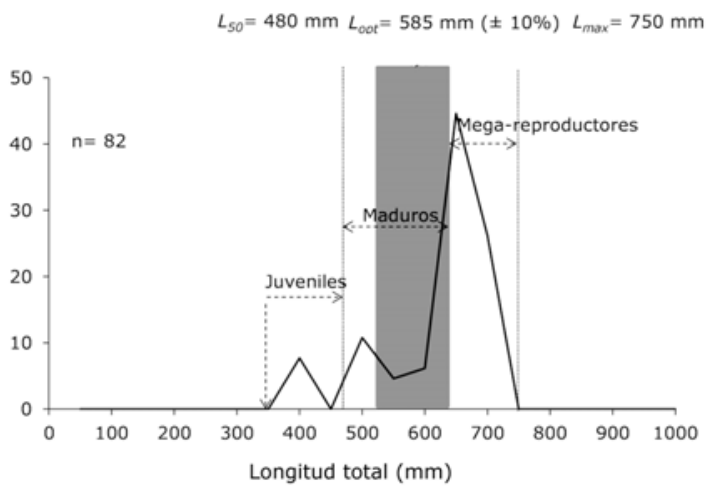

Figura 5. Indicadores de sustentabilidad de Froese (2004) para la distribución de longitud de hembras de curvina golfina Cynoscion othonopterus en el Alto Golfo de California durante la temporada de pesca A) 2008, B) 2013 C) 2014 y D) 2015 . $L_{50}$ : longitud de madurez, $L_{\text {opt }}$ : intervalo de longitud óptima donde se obtiene el máximo rendimiento, $L_{\max }$ : longitud máxima registrada en el temporada / Froese sustainability indicators (2004) for the distribution of female Gulf corvina Cynoscion othonopterus maturity length in Upper Gulf of California during fishing season A) 2008 , B) 2013 C) 2014 and D) 2015. $L_{50}$ : maturity length, $L_{\text {opt }}$ : optimal length interval from which it is obtained the maximum efficiency, $L_{\text {max }}$ : maximum length registered during season

diferentes aberturas de mallas de 12,5 y 15,24 mm (RománRodríguez 2000). Para contrarrestar este problema se implementa en 2008 la estandarización de la luz de malla para la captura de curvina. Se observó que a partir de ese año las longitudes promedio así como sus desviaciones estándar se homogeneizaron. Por lo tanto esta implementación de la regulación de artes tuvo un efecto positivo en la pesquería coincidiendo con Gherard et al. (2013). La única información que existe sobre la evaluación de la luz de malla es de Solana-Sansores et al. (2012) quienes demuestran que solamente el uso de redes de enmalle con 14,6 cm de luz de malla mantiene la pesca incidental de individuos menores a $65 \mathrm{~cm}$ por debajo del $35 \%$ y es la única que cumple con la normativa mexicana. Ahora bien, en un contexto general, los esfuerzos para implementar, evaluar y aplicar estas dos medidas de manejo han sido hasta cierto punto efectivos en el contexto de la precaución para la preservación de la especie. Sin embargo, estas dos medidas están determinadas por la actual longitud de madurez, y como se discutió anteriormente esta medida está sobreestimada debido a los métodos utilizados y requiere de una actualización. Por lo tanto, al estar ligada la longitud de madurez con luz de malla, es necesario que se realice una modificación en diámetro de la red agallera.

Para llevar a cabo una regulación de las pesquerías y permitir que estas continúen siendo explotadas de manera sustentable, se ha establecido en México, a partir de los años 90, el sistema cuotas de captura total en sus esquemas de manejo (INAPESCA 2006). Actualmente la pesquería de curvina golfina se lleva a cabo mediante el esquema de cuotas de capturas, la Norma Oficial Mexicana para la pesca responsable de curvina golfina establece que anualmente el Instituto Nacional de la Pesca (INAPESCA) recomendará la cuota de captura para la pesquería de curvina. Los registros que se tienen en el Diario Oficial de la Federación (DOF) de los años en los 
que se han establecido las cuotas de captura son para las temporadas de pesca 2012-2016 (DOF 2012 $, 2013^{8}, 2014^{9}$, $2015^{10}, 2016^{11}$ ) con una captura total (t) de 2.300 de peso total, 2.700 peso eviscerado ( $P E$ ), 3538,6 PE, 3790 PE y 3602 PE respectivamente. Se destaca que con excepción de la temporada 2014 las cuotas no se respetaron ya que se extrajo más producto de la cuota establecida. Ahora bien, otro aspecto importante es la creciente pesca ilegal no reportada y no regulada. Se estima que esta actividad ilícita en México representa casi la mitad de las capturas reportadas oficialmente (Cisneros-Montemayor et al. 2013). Por otro lado, Ruelas-Peña et al. (2013) y EncisoEnciso (2014) considerando un enfoque precautorio y considerando el mínimo de riesgo para que la pesquería no entre en colapso, proponen no superar las $3.100 \mathrm{y}$ 3.215 t respectivamente de biomasa al máximo rendimiento sostenible. Por ello uno de los puntos para establecer la sustentabilidad en la pesquería de curvina, es respetar los acuerdos que se encuentran vigentes en la norma y plan de manejo, ya que estos acuerdos están basados en la preservación de la especie, el aprovechamiento equitativo del recurso y recomendaciones sustentables por parte de la comunidad científica.

La longitud de madurez sexual es uno de los aspectos más importantes del ciclo biológico de las especies, como también lo es para el manejo de las pesquerías. La longitud de madurez promedio o esperada es generalmente estimada ajustando un modelo logístico a la proporción de hembras por intervalo de longitud. Se puede asumir que el modelo logístico representa una función de densidad de probabilidad y la longitud de madurez corresponde a la longitud donde el 50\% de los individuos de la población están maduros. A la fecha existe escasa información de la longitud de madurez para Cynoscion othonopterus y la información disponible se encuentra en tesis de grado e informes técnicos (lo que comúnmente se conoce como literatura gris). Román-Rodríguez (2000) reportó una $L_{50}$ de $700 \mathrm{~mm}$ calculada mediante observaciones macroscópicas. Acosta-Valenzuela (2008) utilizó el criterio macroscópico de Nikolsky (1963) para diferenciar los organismos maduros e inmaduros, y posteriormente determinó una $L_{50}$ para hembras maduras de $702 \mathrm{~mm}$ a través de mínimos cuadrados mediante la fórmula de King (1995) para datos acumulados. Acosta-Valenzuela (2010) empleó técnicas histológicas y macroscópicas para determinar el estadio de madurez y estimó una $L_{50}$ para machos de 674 y 685 mm para hembras. Por último Gherard et al. (2013) mediante análisis histológicos determinó una longitud media de madurez sexual con respecto a la edad con la que el pez alcanza su madurez sexual obteniendo una $L_{50}$ para machos de 267,5 mm para organismos con 2 años y 294,7 mm para hembras con 2,3 años. En el presente estudio, se encontró que las longitudes de madurez en los periodos analizados no coinciden con los reportados en investigaciones previas. Asimismo, se destaca que todas las $L_{50}$ estimadas en esta investigación están por debajo de la longitud mínima legal establecida en el plan de manejo pesquero de $C$. othonopterus que es de 650 $\mathrm{mm}$ de $L T$. Las diferencias de la longitud de madurez que se reportan para curvina golfina radican en la metodología empleada para estimar esta variable. Mientras que RománRodríguez (2000) y Acosta-Valenzuela (2010) estiman la $L_{50}$ con la frecuencia acumulada que considera la relación que existe entre el número de organismos maduros en una cierta estructura de longitud con el número total de

\footnotetext{
${ }^{7}$ DOF. 2012. Acuerdo por el que se da a conocer el Plan de Manejo Pesquero de Curvina Golfina (Cynoscion othonopterus) del norte del Golfo de California. <http://www.dof.gob.mx/nota_detalle_popup.php?codigo=5276662>

${ }^{8}$ DOF. 2013. Acuerdo por el que se establece la cuota de captura para el aprovechamiento de curvina golfina (Cynoscion othonopterus), en aguas de jurisdicción federal del Alto Golfo de California y Delta del Río Colorado para la temporada 20122013. <http://dof.gob.mx/nota_detalle.php?codigo=5285586\&fecha=24/01/2013>

${ }^{9}$ DOF. 2014. Acuerdo por el que se establece la cuota de captura para el aprovechamiento de curvina golfina (Cynoscion othonopterus), en aguas de jurisdicción federal del Alto Golfo de California y Delta del Río Colorado para la temporada 20132014. <http://dof.gob.mx/nota_detalle.php?codigo=5333652\&fecha=24/02/2014>

${ }^{10}$ DOF. 2015. Acuerdo por el que se suspende temporalmente la pesca comercial mediante el uso de redes de enmalle, cimbras y/o palangres operadas con embarcaciones menores, en el norte del Golfo de California. <http://www.dof.gob.mx/ nota_detalle.php?codigo=5388486\&fecha=10/04>

${ }^{11}$ DOF. 2016. Acuerdo por el que se establece la cuota de captura para el aprovechamiento de curvina golfina (Cynoscion othonopterus), en aguas de jurisdicción federal del Alto Golfo de California y Delta del Río Colorado para la temporada de pesca 2016. <http://dof.gob.mx/nota_detalle.php?codigo=5425994\&fecha=17/02/2016>
} 
organismos maduros, descartando la porción inmaduros creando un error de sobreestimación de la $L_{50}$. Aunado a lo anterior, estas investigaciones ajustaron el modelo con mínimos cuadrados, que si bien este método fue muy utilizado en sus inicios poco a poco fue siendo sustituido por métodos de ajuste más robustos como la máxima verosimilitud. La desventaja de ajustar con mínimos cuadrados es que minimiza el valor del parámetro, no estima la probabilidad del parámetro, no considera una distribución de probabilidad del error y no utiliza desviación estándar del error. En cambio la máxima verosimilitud si toma en cuenta lo antes mencionado, debido a que considera una distribución normal de los datos generando una curva simétrica, es decir a partir del valor medio de $L_{50}$ por lo que la tasa de cambio es monotónicamente hacia ambas asíntotas, la mayor y menor; también maximiza el valor del parámetro, además de utilizar los mínimos cuadrados y la verosimilitud para estimar la probabilidad del parámetro (CervantesHernández et al. 2005). Por otro lado, el modelo binomial propuesto por Brouwer \& Griffiths (2005) se ha puesto a competir con otros modelos de madurez y de acuerdo al criterio de información de Akaike (Akaike 1973), ha resultado ser uno de los mejores modelos para determinar de la longitud de madurez específicamente en hembras (Hernández-Covarrubias et al. 2013, Oviedo-Pérez et al. 2014). Ahora bien, la longitud mínima de captura vigente para curvina golfina está determinada con información del método de frecuencia acumulada con ajuste de mínimos cuadrados, lo que en su momento fue efectivo debido la necesidad de establecer medidas para el aprovechamiento de la especie, aunado a la escasa información sobre métodos robustos que permitieran determinar con más solidez la $L_{50}$. Con base en lo expuesto, la actual longitud de madurez es inadecuada para el manejo de curvina golfina y es necesario actualizarla con métodos más robustos y confiables.

Los indicadores de Froese (2004) están fundamentados en el análisis de los cambios en la frecuencia de longitudes. Estos indicadores son un instrumento útil para el monitoreo de las especies y la recuperación del stock. Asimismo, Froese (2004) demostró la importancia que tienen los peces longevos (mega-reproductores) en la supervivencia de las futuras generaciones de una especie ya que las hembras al ser más grandes y longevas están mejor adaptadas al medio y pueden soportar cambios drásticos del ambiente proporcionando mejores genes a las futuras generaciones. Aunado a lo anterior, hembras más fecundas y el número de huevos por lo general se incrementa con el aumento de la longitud, por lo que las larvas tienen más probabilidades de sobrevivir. Coincidiendo con ello, Trippel (1998) menciona que los mega-reproductores, pueden ser vistos como una reserva de la población para resistir eventos estocásticos. Específicamente para curvina golfina, Acosta-Valenzuela (2010) mediante la estimación de la fecundidad parcial, observó que cuanto más grande sea la hembra se incrementa la viabilidad y el número de huevos. Los indicadores de Froese han sido utilizado para evaluar diversas especies de peces (Froese \& Binohlan 2000, Froese 2004, Mair et al. 2012, Pérez-Lozano \& Aniello 2013) quienes coinciden en que dichos indicadores son efectivos para determinar el grado de explotación de la pesquería, y punto de partida para tomar medidas dirigidas a la sustentabilidad del recurso y propuesta para el manejo de las especies. Por lo anterior se establecieron los criterios de sustentabilidad de Froese (2004) para la pesquería de curvina golfina durante las temporadas de pesca 2008, 2013, 2014 y 2015. Se aporta evidencia de que, a excepción de la temporada de pesca 2014, existe sobreexplotación del recurso con mega-reproductores y juveniles, considerando que Froese (2004) propone que si una pesquería no tiene una longitud máxima de captura, como es el caso de curvina golfina, entonces es tolerable que se tengan en las capturas entre un $30-40 \%$ de megareproductores. Adicionalmente, se establece que en las capturas no deben de existir presencia de juveniles porque esto también indica sobreexplotación. En general se exhibe que la pesquería de curvina está dirigida hacia los organismos de mayor longitud (mega-reproductores) y esto implica indicios de insostenibilidad y potencial riesgo de colapso. Estos resultados difieren de lo reportado por Erisman et al. (2014), quienes utilizaron el promedio del parámetro de crecimiento $\left(L_{\infty}=1.006\right.$, periodo de estudio 2009-2011) de Gherard et al. (2013) para establecer solamente una longitud óptima para el periodo de estudio (1997-2012) y determinar los indicadores de Froese (2004), estableciendo que la pesquería se encontraba saludable. Sin embargo, establecer solo una longitud óptima para 15 años de datos de longitud promedio es incongruente, porque lo apropiado es determinar los indicadores anualmente y compararlos con su $L_{\infty} \mathrm{o} L_{50}$ del mismo año, tal como se realizó en el presente estudio, donde se calculó para cada año la $L_{50}$ mediante métodos confiables y de robustez así como $L_{\text {opt }}$ y estructura de tallas para estimar los indicadores de sustentabilidad. Los resultados arrojan un patrón repetitivo donde se calcula a los megareproductores por encima del $40 \%$ de las capturas así 
como presencia de juveniles por lo que se llegó a la conclusión que la pesquería de curvina golfina presenta indicios de insostenibilidad. Si bien Erisman et al. (2014), aplicó los criterios de sustentabilidad, como dice Froese (2004), no los realizó bien debido a que no calculó la $L_{\text {opt }}$ mediante el uso de $L_{50}$. Si utilizamos como referencia la $L_{\text {opt }}$ que reporta Erisman et al. (2014), de $715 \mathrm{~mm}$ calculada solo con $L_{\max }$ con su respectivo intervalo $\pm 10 \%$ (644-787) y lo comparamos con los datos de longitud total y talla de madurez que ellos mismos reportan para 1997 y 2012 un promedio de $L_{50}$ de $295 \mathrm{~mm}$ comprobaríamos que no se cumple con los indicadores de sustentabilidad descritos por Froese (2004). Todo ello conlleva según los indicadores aquí utilizados a una pesquería no sostenible con riesgo potencial de colapso. Esto ya había sido advertido por Peñaranda-Gonzalez (2013) quien llegó a esta conclusión a partir de un análisis de diversidad genética de curvina golfina cuya evidencia mostró una menor heterocigosidad observada respecto a la esperada y textualmente fue expuesto que 'la deriva genética y la consecuente endogamia, repercuten en la pérdida de la variabilidad genética, disminuyendo la eficacia biológica de los individuos de la población. En su conjunto, estos factores, sumados a la explotación pesquera, no favorecen que la población pueda alcanzar su equilibrio demográfico por lo que la pesca de esta especie podría colapsar'. Winemiller et al. (1996) mencionan que la alteración del ecosistema acuático y la presión por pesca pueden afectar la biomasa del stock de una forma más severa de lo que se tenía contemplado especialmente si estos se combinan con factores los cuales el ser humano no puede controlar como el cambio climático. Welcomme (1992) menciona que las estrategias de conservación espacial y temporal en un plan de manejo son herramientas importantes para ayudar a mantener saludable al stock, en ese sentido las estrategias para mantener saludable a la pesquería de curvina golfina, como periodo de veda y cuota de captura a la fecha han sido de gran importancia aunque insuficientes. Por lo tanto, debido a la presión por pesca y los nuevos retos que actualmente enfrentan los pescadores y las autoridades gubernamentales, es necesario incorporar en la Norma Oficial Mexicana NOM063-PESC-2005 y al plan de manejo pesquero nuevas estrategias que ayude a complementar las medidas de conservación y sustentabilidad actualmente vigentes.

Actualmente la pesquería de curvina golfina muestra indicios de una pesquería no sustentable, sin embargo, existen mecanismos y alternativas para darle seguimiento $\mathrm{y}$ establecer prontas acciones que ayuden a una mejor administración del recurso. Entre estas acciones se puede considerar la variabilidad e identidad genética (RíosMedina 2012, Peñaranda-Gonzalez 2013). Estudios sobre capturas al máximo rendimiento sostenible, proyecciones de captura y la actualización de selectividad de la red debido a la reducción de la talla de madurez. En caso contrario y de seguir esta tendencia el único recurso con permiso de explotación para las localidades del Alto Golfo de California y San Felipe puede llegar a colapsar en un corto o mediano plazo.

\section{Agradecimientos}

JEMM agradece al Consejo Nacional de Ciencia y Tecnología (CONACYT) por la beca otorgada para la realización de sus estudios de posgrado (número de beca 248936). EAAN recibió financiamiento de CONACYT (CB2012-1 Project 178727).

\section{LITERATURA CITADA}

Akaike H. 1973. Information theory and an extension of the maximum likelihood principle. In: Petrov BN \& F Csaki (eds). Second International Symposium on Information Theory, pp. 267-281. Academiai Kiado, Budapest.

Acosta-Valenzuela Y. 2008. Aspectos de la biología reproductiva de la curvina golfina Cynoscion othonopterus en el Alto Golfo de California. Tesis de Licenciatura, Instituto Tecnológico del Valle del Yaqui, Bácum, 65 pp.

Acosta-Valenzuela Y. 2010. Ecología y potencial reproductivo de la curvina golfina Cynoscion othonopterus (Jordan \& Gilbert 1882) en el Alto Golfo de California y Delta del Rio Colorado. Tesis de Maestría, Instituto Tecnológico de Guaymas, Guaymas, 122 pp.

Barbarino A, D Taphorn \& KO Winemiller. 1998. Ecology of coporo Prochilodus mariae (Characiformes: Prochilodontidae) and status of annual migrations in western Venezuela. Environmental Biology of Fishes 53: 33-46.

Brouwer SL \& MH Griffiths. 2005. Reproductive biology of carpenter seabream (Argyrozona argyrozona) (Pisces: Sparidae) in a marine protected area. Fishery Bulletin 103: 258-269.

Campoy JD. 1999. Análisis de la situación pesquera en la reserva de la biósfera Alto Golfo de California y Delta del río Colorado, 5 pp. INE/SEMARNAT, México.

Cervantes-Hernández P, A Flores-Gómez \& B SánchezMeraz. 2005. Mínimos cuadrados vs. verosimilitud. Ciencia y Mar 9(27): 41-45.

Cisneros-Montemayor AM, MA Cisneros-Mata, S Harper \& D Pauly. 2013. Extent and implications of IUU in Mexico's marine fisheries. Marine Policy 39: 283-288. 
Doode S \& P Wong. 2001. El Golfo de California: surgimiento de nuevos actores sociales, ambientalismo y región, México. Estudios Sociales 11: 25-56.

Enciso-Enciso C. 2014. Evaluación de la pesquería de curvina golfina Cynoscion othonopterus (Gilbert \& Jordan 1882) en el Alto Golfo de California. Tesis de Maestría, Facultad de Ciencias del Mar, Universidad Autónoma de Sinaloa, Mazatlán, 65 pp. <http://www.uv.mx/personal/gagalindo/ files/2015/01/tesis-cuvina-golfina-Cenciso.pdf>

Erisman BE, MAApel, AD MacCall, MJ Román-Rodríguez \& R Fujita. 2014. The influence of gear selectivity and spawning behavior on a data-poor assessment of a spawning aggregation fishery. Fisheries Research 159: 75-87.

FAO. 1995. Código de conducta para la pesca responsable, 46, pp. FAO, Roma. <http://www.fao.org/docrep/005/V9878S/ V9878S00.htm>

FAO. 2000. Indicadores para el desarrollo sostenible de la pesca de captura marina. FAO Orientaciones Técnicas para la Pesca Responsable 8: 1-68, FAO, Roma. <http:// www.fao.org/3/a-x3307s.html>

Froese R. 2004. Keep it simple: three indicators to deal with overfishing. Fish and Fisheries 5: 86-91.

Froese R \& C Binohlan. 2000. Empirical relationships to estimate asymptotic length, length at first maturity and length at maximum yield per recruit in fishes, with a simple method to evaluate length frequency data. Journal of Fish Biology 56: 758-773.

García S, D Staples \& J Chesson. 2000. The FAO guidelines for the development and use of indicators for sustainable development of marine capture fisheries and an Australian example of their application. Ocean \& Coastal Management 43(7): 537-556.

Gherard K, BE Erisman, K Rowell \& LG Allen. 2013. Growth, development, and reproduction of Gulf corvina (Cynoscion othonopterus). Bulletin of the Southern California Academy Sciences 112(1): 1-18. <doi:10.3160/ 0038-3872-112.1.1>

Haddon M. 2001. Modelling and quantitative methods in fisheries, 406 pp. Chapman \& Hall/CRC, Boca Raton.

Hernández-Covarrubias V, JL Patiño-Valencia \& $\mathbf{H}$ Aguirre-Villaseñor. 2013. Inferencia multimodelo: cálculo de la talla media de madurez del ostión de roca Striostrea prismatica en Nayarit, México. Ciencia Pesquera 22(1): 11-18.

INAPESCA. 2006. Sustentabilidad y pesca responsable en México: Evaluación y manejo, 544 pp. Instituto Nacional de Pesca, Secretaría de Agricultura, Ganadería, Desarrollo Rural, Pesca y Alimentación, México. <http:// www.inapesca.gob.mx/portal/documentos/publicaciones/ pelagicos/libro_Rojo.pdf>

King M. 1995. Reproduction and recruitment. In: King M (ed). Fisheries biology, assessment and management, pp. 151-165. Fishing News Books, Oxford.
Mair JM, R Cipriani, HM Guzman \& D Usan. 2012. Fishery of the Green Jack Caranx caballus (Osteichytes: Carangidae) in Las Perlas Archipelago, Pacific Panama. Revista de Biología Tropical 60(30): 1271-1288.

Nikolsky GV. 1963. The ecology of fishes, 351 pp. Academic Press, New York.

Oviedo-Pérez JL, H Zea-De la Cruz, DH Aguirre-Villaseño, C Meiners-Mandujano, L Jiménez-Badillo \& $\mathbf{L}$ González-Ocaranza. 2014. Talla de madurez sexual del tiburón Rhizoprionodon terraenovae en Veracruz, México. Ciencia Pesquera 22(1): 37-45.

Pauly D. 1983. Algunos métodos simples para la evaluación de recursos pesqueros tropicales. FAO Documento Técnico de Pesca 234: 1-49.

Peñaranda-Gonzalez LV. 2013. Caracterización genéticopoblacional basada en marcadores moleculares tipo microsatélites en la corvina golfina (Cynoscion othonopterus) del Alto Golfo de California. Tesis de Maestría, Universidad Autónoma de Baja California, Ensenada, 65 pp. <https://goo.gl/5zqDuK>

Pérez-Lozano A \& B Aniello. 2013. Parámetros poblacionales de los principales recursos pesqueros de la cuenca del río Apure, Venezuela (2000-2003). Latin American Journal of Aquatic Research 4(3): 447-458.

Rice JC \& MJ Rochet. 2005. A framework for selecting a suite of indicator for fisheries management. Journal of Marine Science 62: 516-527.

Ríos-Medina K. 2012. Diversidad genética de la corvina golfina (Cynoscion othonopterus) en la Reserva de la Biosfera del Alto Golfo de California y Delta del Río Colorado. Tesis de Maestría, Universidad Autónoma de Baja California, Ensenada, 103 pp. <https://goo.gl/MKwKXs>

Rodríguez-Quiroz G, EA Aragón-Noriega, W ValenzuelaQuiñonez \& HM Esparza-Leal. 2010. Artisanal fisheries in the conservation zones of the upper Gulf of California. Revista de Biología Marina y Oceanografía 45: 89-98.

Román-Rodríguez MJ. 2000. Estudio poblacional del Chano Norteño, Micropogonias megalops y la Curvina Golfina Cynoscion othonopterus (Gilbert \& Jordan 1882) (Pisces: Sciaenidae), especies endémicas del Alto Golfo de California, México. Instituto del Medio Ambiente y Desarrollo Sustentable del Estado de Sonora. Hoja de cálculo SNIBCONABIO proyecto No. L298. CONABIO, Distrito Federal, México, 143 pp. <http://www.conabio.gob.mx/ institucion/proyectos/resultados/InfL298.pdf>

Román-Rodríguez MJ \& J Barrera-Guevara. 2006. Diagnóstico y elaboración del plan de manejo de la curvina golfina (Cynoscion othonopterus, Jordan y Gilbert, 1881) en el Alto Golfo de California, México. Informe final para la SAGARPA, Gobierno del Estado de Sonora, Comisión de Ecología y Desarrollo Sustentable del Estado de Sonora, Hermosillo, 137 pp. 
Ruelas-Peña JH, C Valdez-Muñoz \& EA Aragón-Noriega. 2013. La pesquería de la curvina golfina y las acciones de manejo en el Alto Golfo de California, México. Latin American Journal of Aquatic Research 4(3): 498-505.

Solana-Sansores LR, I Dicante, L Luna \& R VillaseñorTalavera. 2012. Selectividad de redes para capturar curvina golfina (Cynoscion othonopterus) en el Alto Golfo de California, México. Hidrobiológica 22(2): 132-141.

Trippel EA. 1998. Egg size and viability and seasonal offspring production of young Atlantic cod. Transactions of the American Fisheries Society 127: 339-359.
Venzon DJ \& SH Moolgavkar. 1988. A method for computing profile-likelihood-based confidence intervals. Journal of the Royal Statistical Society 37(1): 87-94.

Welcomme RL. 1992. Pesca fluvial. FAO Documento Técnico de Pesca 262: 1-303. <http://www.fao.org/docrep/003/ T0537S/T0537S00.HTM>

Winemiller K, C Marrero \& D Taphorn. 1996. Perturbaciones causadas por el hombre en las poblaciones de peces de los llanos y del pie de monte andino de Venezuela. Biollania 12: 13-37.

Recibido el 27 de diciembre de 2016 y aceptado el 24 de abril de 2018

Editor: Claudia Bustos D. 